\title{
El impacto de redes de colaboración en la innovación tecnológica en empresas
}

\section{The impact of collaboration networks on technological innovation in firms}

Dr. Javier Del Carpio-Gallegos es profesor e investigador de la Universidad ESAN (Perú) (jdelcarpio@esan.edu.pe) (https:// orcid.org/0000-0001-6050-5754)

Dr. Francesc Miralles es profesor e investigador de La Salle-Universidad Ramon Llull (España) (francesc.miralles@ salle.url.edu) (https://orcid.org/0000-0002-5251-5423)

\begin{abstract}
Resumen
Las empresas manufactureras latinoamericanas deben desarrollar innovaciones para ser competitivas. Por ello, esta investigación tiene dos objetivos: primero, analizar cómo se relacionan las conexiones con clientes, proveedores, competidores e institutos de investigación con la capacidad de absorción, y, segundo, demostrar la relación existente entre la capacidad de absorción y la innovación tecnológica. Se desarrolló un modelo teórico para mostrar la relación entre las variables, a las cuales se les aplicó el método de ecuaciones estructurales, utilizando el software AMOS, a una muestra de 1098 empresas manufactureras peruanas que participaron en la segunda encuesta nacional de innovación realizada en 2015. Este estudio contribuye a la literatura sobre cómo las empresas se relacionan con las redes de colaboración para mejorar la capacidad de innovación, de esta manera obtienen conocimiento aplicando el enfoque de innovación abierta entrante, este conocimiento procesado a través de la capacidad de absorción de la empresa mejorará su capacidad de innovación. Las empresas manufactureras de intensidad tecnológica baja o media-baja realizan inversiones en maquinaria, hardware y software y tienen más capacidad para realizar innovaciones de productos y procesos. Las innovaciones en producto permiten a las empresas mantener o incrementar su posición en el mercado o tener clientes más satisfechos, al tiempo que realizan innovaciones en los procesos para reducir sus costos operativos o ser más eficientes.
\end{abstract}

\begin{abstract}
Latin American manufacturing firms must develop innovations to be competitive. For this reason, this research has two objectives: first, to analyze how connections with customers, suppliers, competitors, and research institutes are related to absorptive capacity, and, second, to demonstrate the existing relationship between absorptive capacity and technological innovation. A theoretical model was developed to show the relationship among the variables. The structural equation modelling was applied through AMOS software to a sample of 1,098 Peruvian manufacturing firms that participated in the second national innovation survey carried out in 2015. This study contributes to the literature on how firms relate with collaboration networks to improve their innovation capacity. In this way, firms obtain knowledge by applying the inbound open innovation approach. This knowledge is processed through each firm's absorptive capacity and will improve its innovation capacity.

Low or medium-low tech manufacturing firms that make investments in machinery, hardware, and software are more able to carry out product and process innovations. Product innovations allow firms to maintain or increase their position in the market and have more satisfied customers, while process innovations reduce their operating costs and make them more efficient.
\end{abstract}

\section{Palabras clave I keywords}

Redes, colaboración, capacidad, absorción, innovación, emergentes, manufactura, modelo.

Networks, collaboration, capacity, absorption, innovation, emerging, manufacturing, model.

Cómo citar: Del Carpio-Gallegos J., y Miralles, F. (2021). El impacto de redes de colaboración en la innovación tecnológica en empresas. Retos Revista de Ciencias de la Administración y Economía, 11(22), pp. 315-331. https://doi.org/10.17163/ret.n22.2021.08 


\section{Introducción}

La innovación es un tema que ha captado la atención de académicos y directivos. A través del desarrollo de la capacidad de innovación, las empresas pueden volverse más competitivas (Coccia, 2017), alcanzar mayores niveles de exportación (Love \& Roper, 2015) e introducir productos y servicios al mercado que satisfagan las demandas de sus clientes (King \& Baatartogtokh, 2015). En este sentido, las empresas buscan socios con los que conectarse y, de esta forma, mejorar su capacidad de innovación (Scuotto et al., 2017).

Debido a que las conexiones con socios comerciales como clientes, proveedores, competidores, universidades o asociaciones industriales ayudan a las empresas a desarrollar innovaciones (Lai et al., 2014) y debido a los beneficios que puede generar una empresa con esta capacidad, es necesario realizar estudios que muestren cómo las conexiones con socios clave se relacionan con la capacidad de absorción, que es una capacidad dinámica (Eisenhardt \& Martin, 2000), útil para las empresas que desean recopilar conocimiento de otras fuentes con el fin de fortalecer su nivel de capacidad de innovación tecnológica.

Las redes de colaboración llaman la atención porque las empresas que son capaces de crear la mayor cantidad de conexiones a través de redes de colaboración también son más capaces de desarrollar su capacidad de innovación (Marrocu et al., 2013). Sin embargo, al mismo tiempo, los gerentes reconocen que las conexiones con los clientes brindan ideas para nuevos productos, así como las conexiones con proveedores y universidades brindan conocimiento para desarrollar innovaciones tecnológicas y las conexiones con competidores brindan ideas y la motivación para seguir innovando (Baker et al., 2016).

Por otro lado, Cohen y Levinthal (1990, p.128) especifican que "la capacidad de absorción es la habilidad de una empresa para reconocer el valor de información nueva, externa, asimilarla y aplicarla" para fortalecer su capacidad de innovación tecnológica. En efecto, abundantes estudios muestran cómo la innovación tecnológica evoluciona con mayor rapidez en presencia de capacidad de absorción (Martín-de Castro, 2015), pero también es importante mencionar que estos estudios se enfocan en empresas de alta tecnología (Tzokas et al., 2015), es decir, aquellas empresas que dedican importantes recursos financieros a realizar investigación y desarrollo en economías desarrolladas. Estos estudios ignoran, en gran medida, a empresas de economías emergentes que, a pesar de no dedicar grandes cantidades de recursos a la investigación y el desarrollo, también realizan innovaciones tecnológicas, invirtiendo en la compra de maquinaria, hardware y software (Goedhuys et al., 2014).

La literatura a revisar es abundante cuando el fenómeno que se estudia es la capacidad de innovación de empresas con mayor intensidad tecnológica en economías desarrolladas (Conte \& Vivarelli, 2014). No obstante, pocos estudios se han centrado en las economías emergentes y las empresas de tecnología baja y media-baja que las componen; de hecho, pocos investigadores se han centrado en la región latinoamericana (Del Carpio \& Miralles, 2018; Ponce-Espinosa et al., 2017; Zapata-Rotundo \& Hernández-Arias, 2018; Romero et al., 2021). Sin embargo, las empresas manufactureras de baja tecnología de estos países dedican un gran esfuerzo a desarrollar la capacidad de innovación, lo que contribuye a que sean más competitivas en el mercado mundial.

Este estudio plantea la siguiente pregunta de investigación: ¿Qué factores influyen en la capacidad de innovación de las empresas de baja tecnología en las economías emergentes? Sus objetivos específicos son: primero, analizar cómo se relacionan las 
conexiones con clientes, proveedores, competidores e institutos de investigación con la capacidad de absorción (Nicotra et al., 2014), y segundo, demostrar la relación existente entre capacidad de absorción e innovación tecnológica (Del Carpio \& Miralles, 2018). La información utilizada en esta investigación corresponde a la segunda encuesta de innovación de la industria manufacturera realizada en Perú en 2015. La unidad de análisis es la empresa manufacturera de baja o media-baja tecnología que participó en dicha encuesta.

La estructura de este estudio es: después de esta introducción, se presenta el marco teórico y se formulan las hipótesis. La tercera sección explica la metodología, describe los datos, define las variables de estudio y explica los procedimientos estadísticos utilizados para analizar los datos. La cuarta sección muestra los resultados, y luego la quinta sección presenta la discusión de los resultados. Finalmente, se presentan las conclusiones, limitaciones del estudio y futuras líneas de investigación.

\subsection{Marco teórico y formulación de hipótesis}

Este estudio se realiza bajo la teoría de las capacidades dinámicas, las cuales son "un conjunto de procesos específicos e identificables como el desarrollo de productos, la toma de decisiones estratégicas y las alianzas" (Eisenhardt \& Martin, 2000, p. 1105). Es importante señalar que "el enfoque de capacidades dinámicas se ha construido sobre las ideas de Schumpeter" (Breznik \& Hisrich, 2014, p. 374).

\subsubsection{Redes de colaboración y capacidad de absorción}

Las redes de colaboración se han vinculado al desarrollo de la capacidad de absorción de las empresas manufactureras (Agramunt et al., 2020). Tsai (2001) hace esta observación en su estudio sobre productores de alimentos, encontrando un vínculo entre la posición de estas empresas en sus respectivas redes de colaboración y su nivel de capacidad de absorción. Además, Spithoven et al. (2010) indican que las empresas que pertenecen a industrias maduras desarrollan una capacidad de absorción para implementar las llamadas "prácticas de innovación abierta"; es decir, interactúan con clientes, proveedores y otros actores para que estos actores les brinden la información necesaria para mejorar su capacidad de innovación. Por otro lado, Cantner y Joel (2011) indican que la interacción con diferentes actores pertenecientes a redes de colaboración conduce a la generación de conocimiento que, con la ayuda de la capacidad de absorción, conduce a la mejora de la capacidad de innovación de la empresa. En esta línea, Hurmelinna-Laukkanen et al. (2012) sostienen que la capacidad de absorción y la estabilidad de las redes colaborativas ayudan a las empresas a mejorar su capacidad de innovación. Las relaciones en estas redes colaborativas pueden ser formales, a través de la firma de contratos, o informales, basadas en la confianza mutua de los participantes, ya que lo que buscan es compartir conocimientos.

Además, es importante señalar que Najafi-Tavani et al. (2013) observaron un vínculo entre la capacidad de absorción, los desarrollos de innovación de productos y la participación de los proveedores. Dos de los factores que dependen de la participación de los proveedores son el intercambio de información técnica y las sugerencias que los proveedores pueden aportar en las primeras etapas del desarrollo de nuevos productos. Además, Sáenz et al. (2014) mencionan la forma crucial en que la relación proveedor-comprador se ve afectada por la capacidad de absorción. Los gerentes no solo quieren ser cuidadosos en la selección de sus proveedores; también deben desarrollar la capacidad de absorción para mejorar su capacidad de innovación. También es importante mencionar que Scuotto et al. (2017) quienes encontraron que cuando las 
empresas tienen niveles más altos de capacidad de absorción, su interacción con redes colaborativas aumenta como resultado su capacidad de innovación.

Estos argumentos han permitido formular la siguiente hipótesis:

H1: Las redes de colaboración están relacionadas con la capacidad de absorción en empresas de intensidad tecnológica baja y media-baja.

\subsubsection{Capacidad de absorción e innovación de productos}

La capacidad de absorción permite a las empresas mejorar su capacidad de innovación con respecto a sus productos (Coronado-Medina et al., 2020). Murovec y Prodan (2008) analizaron la fuerte relación entre la capacidad de absorción de las empresas eslovenas y su capacidad para desarrollar innovaciones de productos. Por otra parte, Zhou y Wu (2010) indican que las empresas interactúan con sus clientes, proveedores, competidores y otros para obtener información para ser procesada a través de la capacidad de absorción y, de esta forma, realizar innovaciones de producto. Asimismo, Huang y Rice (2012) argumentan que la capacidad de absorción es un requisito indispensable para las empresas que desean realizar innovaciones de producto.

Por otro lado, Ritala y Hurmelinna-Laukkanen (2013) se centran en la colaboración entre las empresas y sus competidores, destacando el papel de la capacidad de absorción en la asimilación del conocimiento y su conversión en innovaciones de producto, así como su papel en la protección de las innovaciones de las empresas para no sean imitadas por los competidores. Es importante mencionar que, además, Moilanen et al. (2014) analizaron el papel mediador de la capacidad de absorción entre los flujos de conocimiento que provienen de la interacción de las empresas con sus redes colaborativas y su desempeño en innovación. Martínez-Senra et al. (2015) verificaron empíricamente que las empresas que enfrentan un régimen de apropiabilidad sólido, es decir, firmas que pueden proteger su propiedad intelectual, son más capaces de desarrollar innovaciones de producto a pesar de tener un bajo nivel de capacidad de absorción.

Así, se formula la siguiente hipótesis:

H2: La capacidad de absorción está relacionada con la innovación de productos en empresas de intensidad tecnológica baja y media-baja.

\subsubsection{Capacidad de absorción e innovación de procesos}

La capacidad de absorción está relacionada con la capacidad de innovación de procesos (Aliasghar et al., 2020) y, en este sentido, Murovec y Prodan (2008) analizaron la fuerte relación que existe entre la capacidad de absorción de las empresas eslovenas y su capacidad para desarrollar innovaciones de procesos. Asimismo, Segarra-Blasco y Arauzo-Carod (2008) señalan que las empresas españolas presentan bajos niveles de capacidad de absorción y por ello buscan convenios con universidades y centros de investigación para mejorar su capacidad de desarrollar innovaciones de procesos. Hervas-Oliver et al. (2016) creen que es más probable que las empresas desarrollen innovaciones de proceso cuando tienen niveles más altos de capacidad de absorción y capacidad de innovación organizacional.

Finalmente, Jespersen, Rigamonti et al. (2018) demostraron cómo es más probable que las innovaciones de procesos sean desarrolladas por empresas capaces de sintetizar el conocimiento de sus socios. Bayona-Saez et al. (2017) encontraron que la capacidad de absorción es más determinante de la innovación de productos que de la innovación de procesos. Adicionalmente, Del Carpio y Miralles (2018), al analizar empresas manufactureras peruanas de baja tecnología, identificaron un vínculo entre innovación tecnológica, es decir, innovación de productos y procesos, y capacidad de absorción. 
Con base en lo anterior, se formula la tercera hipótesis:

H3: La capacidad de absorción está relacionada con la innovación de procesos en empresas de intensidad tecnológica baja y media-baja.

\subsubsection{Adquisición tecnológica e innovación de productos}

Varios estudios indican que la adquisición tecnológica, es decir, la adquisición de software, hardware y maquinaria por parte de las empresas, ayuda a aumentar la capacidad de innovación de las empresas (Frigon et al., 2020). En esta misma línea, Conte y Vivarelli (2014) analizaron los datos de una encuesta de innovación de más de 3000 empresas italianas y encontraron que la adquisición de maquinaria tenía una relación positiva con la innovación de procesos, pero no con la innovación de productos. Además, Filippetti (2011), utilizando datos de la encuesta europea Innobarometer 2009, encontró que las empresas que se centraban en la reducción de costes y eran, en general, empresas de baja o media-baja tecnología tenían más probabilidades de desarrollar innovaciones de productos. Por otro lado, Goedhuys y Veugelers (2012) mostraron que las empresas brasileñas mejoraron su capacidad tecnológica a través de la adquisición de maquinaria y equipos que les ayudaron a realizar innovaciones de productos y procesos.

En esta línea, Pellegrino et al. (2012), utilizando la base de datos de la tercera encuesta de innovación comunitaria de la industria italiana, encontraron que la adquisición de maquinaria, software y hardware tiene una relación positiva con la innovación de productos para empresas jóvenes, pero no para empresas maduras. Aún más importante, Liao y Barnes (2015) encontraron que la información de los proveedores de maquinaria y equipo hacía que la capacidad de innovación de productos fuera más flexible en las empresas que tenían menos de 250 empleados. Sin embargo, Frank et al. (2016) indican que aun cuando la inversión en adquisición de maquinaria es una de las actividades de innovación que realizan con mayor frecuencia las empresas brasileñas, esta actividad no se refleja en sus resultados de innovación.

Según lo mencionado anteriormente, se formula la cuarta hipótesis:

H4: La adquisición de maquinaria, hardware y software está relacionada con la innovación de productos en empresas de intensidad tecnológica baja y media-baja.

\subsubsection{Adquisición tecnológica e innovación de procesos}

La adquisición tecnológica y su relación con la innovación de procesos ha sido analizada en diferentes circunstancias (Murmura et al., 2021). Asimismo, Reichstein y Salter (2006) analizaron una encuesta de innovación de empresas inglesas y encontraron que la innovación de procesos está relacionada con la incorporación de nueva maquinaria. Rouvinen (2002), al analizar la información de una encuesta de innovación finlandesa, encontró que la adquisición de maquinaria y las conexiones con los proveedores de maquinaria facilitó la innovación de procesos. Además, Vaona y Pianta (2008), al analizar la segunda encuesta de innovación comunitaria de la industria manufacturera en ocho países europeos, encontraron que la adquisición de maquinaria, hardware y software tiene una relación positiva con la innovación de procesos, independientemente del tamaño de la empresa.

Varios años después, Piening y Salge (2015), analizando la industria alemana, encontraron que las empresas de baja tecnología, a través de la compra de maquinaria, hardware y software, adquieren conocimientos que les ayudaron a desarrollar innovaciones en los procesos. Del mismo modo, Hervas-Oliver et al. (2016), al analizar la encuesta española de innovación comunitaria de 2006, encontraron que las empresas que llevan a cabo innovaciones de procesos también realizan adquisiciones de maqui- 
naria, hardware y software. Además, Martino et al. (2017) analizaron empresas de la industria italiana del aceite de oliva y encontraron que aquellas empresas que realizaban inversiones en maquinaria, hardware y software estaban en mejores condiciones para llevar a cabo innovaciones de procesos.

Según estos hallazgos, se formula la quinta hipótesis:

H5: La adquisición de maquinaria, hardware y software está relacionada con la innovación de procesos en empresas de intensidad tecnológica baja y media-baja.

\subsubsection{Innovación de procesos e innovación de productos}

La innovación de procesos y su impacto en la innovación de productos en la industria manufacturera se han analizado varias veces en el pasado. Por ejemplo, Gunday (2011) investigó a 184 empresas manufactureras turcas y encontró que cuanto mayores son los niveles de innovaciones de procesos, mayores son los niveles de innovaciones de productos. De la misma manera, Hassan et al. (2013) encontraron que la innovación de procesos tiene una relación positiva con la innovación de productos. Asimismo, Roldan y Bastos (2019), quienes estudiaron más de 230 empresas orientadas a la tecnología en Brasil, identificaron que la innovación de productos y la innovación de procesos están relacionadas.

Adicionalmente, Camisón y Villar-López (2014) encontraron que la innovación de procesos reduce costos y cambia la forma en que se generan nuevos productos, lo que favorece la innovación de productos. Ballot et al. (2015), al analizar los datos de la cuarta encuesta de innovación comunitaria, que incluyó empresas francesas e inglesas, encontraron complementariedad entre la innovación de procesos y productos, ya que la introducción de nuevos productos también exige cambios en los procesos de producción. Además, Doran (2012) realizó una revisión de la literatura sobre la complementariedad entre la innovación de procesos y productos y encontró que muchos autores sí los consideraban complementarios; es decir, estos autores creían que la innovación de procesos conduce a la innovación de productos y viceversa.

Con base en lo anterior, formulamos la sexta hipótesis:

H6: La innovación de procesos está relacionada con la innovación de procesos en empresas de intensidad tecnológica baja y media-baja.

\subsubsection{El papel mediador de la capacidad de absorción en la relación entre redes de colaboración e innovación tecnológica}

El papel mediador de la capacidad de absorción ha motivado muchos estudios, incluida la investigación de Tsai (2001), quien encontró que la interacción entre la capacidad de absorción y las redes de colaboración favoreció el desarrollo de la capacidad de innovación de las empresas. En esta misma línea, Grimpe y Sofka (2009) argumentan que la capacidad de absorción contribuye a la mejora del desempeño de innovación de las empresas si son capaces de conectarse con clientes y competidores, lo que les da acceso a información de mercado, o con proveedores y universidades, que les proporciona información relacionada con la tecnología. Además, Liao et al. (2010) mencionan que la relación entre la capacidad de innovación y la adquisición de conocimiento está mediada por la capacidad de absorción. Además, indican que la adquisición de conocimientos se puede facilitar a través de las relaciones con proveedores, clientes, universidades e incluso competidores.

Por otro lado, Kostopoulos et al. (2011), interesados en cómo la capacidad de absorción media el desempeño de la innovación y el flujo de conocimiento, destacaron que el flujo de conocimiento es el resultado de la interacción con agentes externos a la empresa. Además, Moilanen et al. (2014) analizaron pequeñas empresas que invierten 
poco o nada en investigación y desarrollo y encontraron que su capacidad de absorción cumplía un papel mediador completo en la relación entre el flujo de conocimiento y el desempeño de innovación de esas empresas.

Por ello, se propone la siguiente hipótesis:

H7a: La capacidad de absorción media la relación entre las redes de colaboración y la innovación de productos.

H7b: La capacidad de absorción media la relación entre las redes de colaboración y la innovación de procesos.

\section{Metodología}

\subsection{Datos}

Para esta investigación, se utilizaron datos de la Encuesta Nacional (de Perú) de Innovación de 2015 en la Industria de Manufactura. La recopilación de datos tuvo lugar durante el período de referencia 2012-2014; la muestra representativa fue de 1452 empresas grandes, medianas y pequeñas; sin embargo, para los propósitos de este estudio, solo se consideran 1098 empresas manufactureras de baja y media-baja tecnología debido a que el Perú, como país emergente, tiene un mayor porcentaje de empresas de baja y media-baja tecnología.

Para analizar el modelo de investigación de la Figura 1, se utilizó un modelo de ecuaciones estructurales para evaluar las hipótesis.

\section{Figura 1. Modelo propuesto}

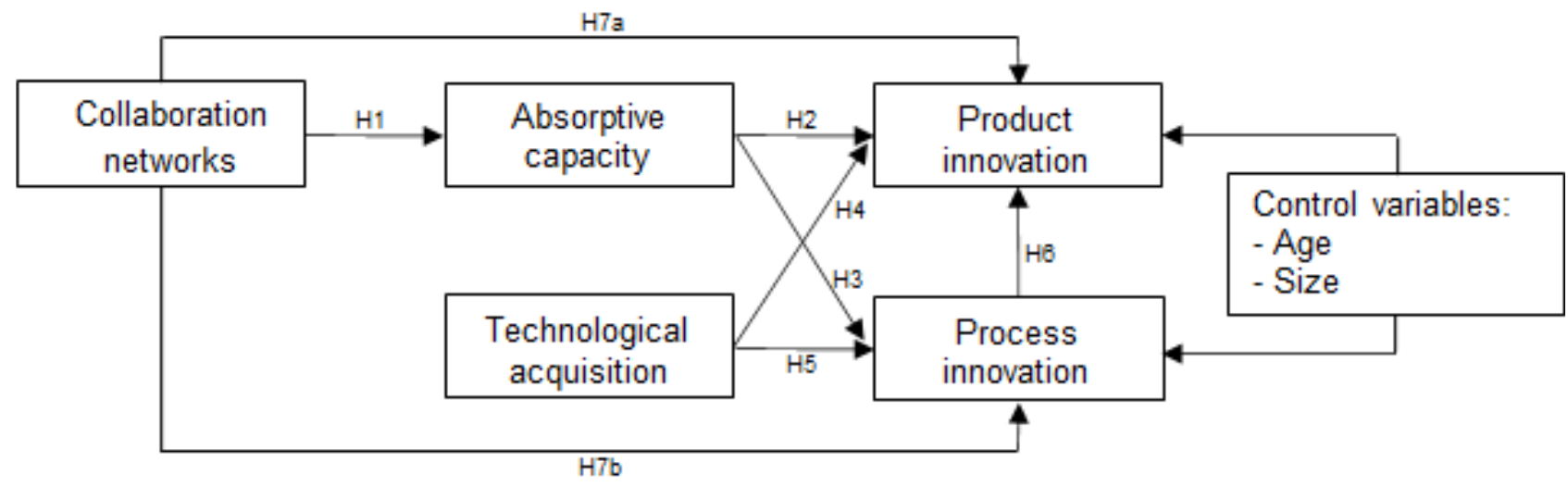

\subsection{Medición de las variables}

\subsubsection{Variables dependientes}

Innovación de producto

La innovación de productos es el resultado de respuestas dicotómicas a la siguiente pregunta: ¿ha podido la empresa introducir en el mercado lo siguiente: un bien nuevo, un servicio nuevo, un bien mejorado significativamente o un servicio mejorado significativamente (Gronum, 2012)?

Innovación de procesos

La innovación de procesos es el resultado de respuestas dicotómicas a la siguiente pregunta: ¿la empresa fue capaz de introducir lo siguiente: un nuevo medio de produc- 
ción de bienes o prestación de servicios; un nuevo método de logística, distribución o despacho de insumos, bienes o servicios; una nueva actividad de apoyo a la producción, como mantenimiento o sistemas de adquisición, contabilidad o TI; un método de producción de bienes o prestación de servicios significativamente mejorado; un método significativamente mejorado para la logística, distribución o despacho de insumos, bienes o servicios; una actividad de apoyo a la producción significativamente mejorada, como sistemas de mantenimiento o adquisiciones, contabilidad o informática (Gronum, 2012)?

\subsubsection{Variables independientes}

Redes de colaboración

Según Nieto y Santamaría (2007), las redes de colaboración se miden por cómo conectan a la empresa con los siguientes agentes o instituciones: universidades, proveedores, clientes, competidores, asociaciones industriales y consultores. Las variables son dicotómicas (SÍ o NO), según la conexión de estos actores con la empresa.

Capacidad de absorción

La capacidad de absorción se mide teniendo en cuenta las propuestas de Escribano et al. (2009). En este sentido, se proponen tres variables: gastos internos de investigación y desarrollo tecnológico; gastos relacionados con la formación para actividades de innovación; y si la compañía cuenta con un departamento de investigación y desarrollo. Todas las variables son dicotómicas (SÍ o NO).

\section{Adquisición tecnológica}

La adquisición tecnológica se mide según las propuestas de Escribano et al. (2009). Se definen tres variables: compras de activos de capital, compras de hardware y compras de software. Todas las variables tienen una transformación logarítmica.

\subsubsection{Variables de control}

El tamaño de la empresa es una variable que se mide por el logaritmo del número total de empleados, según Caloghirou et al. (2004) y Schoenmakers y Duysters (2006), y la edad de la empresa se mide como el número de años (expresado en logaritmo) desde su fundación hasta la actualidad.

\section{Método estadístico}

Se utilizó el software AMOS versión 27 para realizar la ecuación estructural de covarianza de dos pasos para crear un modelo de estimación, de acorde a Medrano y Muñoz-Navarro (2017). En primer lugar, se estimó el modelo de medición cuando se determinó la relación entre los indicadores y el constructo latente mediante Análisis Factorial Confirmatorio (AFC). En segundo lugar, se realizó la estimación del modelo estructural, en el cual se obtuvieron las relaciones entre los constructos utilizando los coeficientes y el nivel de significancia estadística.

\section{Resultados}

\subsection{Modelo de medición}

Los datos de la investigación fueron analizados y presentados utilizando indicadores de confiabilidad y convergencia. 
En términos de confiabilidad, los valores de confiabilidad compuesta (CC) medidos son superiores a 0,7 , y todos ellos tienen valores de varianza media extraída (VME) superiores a 0,5. En cuanto a la multicolinealidad, se controla el factor de inflación de la varianza (FIV), con niveles menores a 5. Con base en los resultados de los indicadores de la Tabla 1, fue posible realizar el modelo estructural. Además, todos los valores de $\mathrm{R}^{2}$ fueron aceptados en las variables endógenas, lo que indica el buen efecto del modelo de las empresas de baja tecnología. Finalmente, la Tabla 2 revela que todas las variables alcanzan validez discriminante, tomando en cuenta el Criterio de Fornell-Larcker (1981).

Tabla 1. Indicadores de fiabilidad y validez

\begin{tabular}{|l|r|r|r|r|}
\hline \multicolumn{1}{|c|}{ Variable latente } & FC & VME & VIF & \multicolumn{1}{c|}{$\boldsymbol{R}^{\mathbf{2}}$} \\
\hline Redes de colaboración & 0.847 & 0.535 & 1.082 & \\
\hline Capacidad de absorción & 0.792 & 0.574 & 1.307 & 0.520 \\
\hline Adquisición tecnológica & 0.826 & 0.616 & 1.291 & \\
\hline Innovación de producto & 0.860 & 0.633 & & 0.230 \\
\hline Proceso de innovación & 0.882 & 0.561 & & 0.434 \\
\hline Valores referenciales & $>0.7$ & $>0.5$ & $<5$ & \\
\hline
\end{tabular}

FC, fiabilidad compuesta; VME, varianza media extraída; VIF, factor de inflación de la varianza.

Fuente: Software SPSS y AMOS.

Tabla 2. Validez discriminante

\begin{tabular}{|c|c|c|c|c|c|}
\hline & 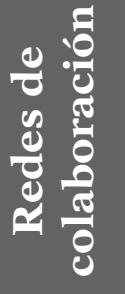 & 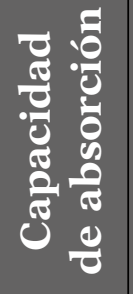 & 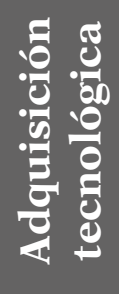 & 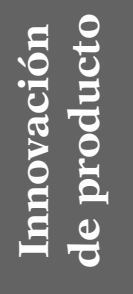 & 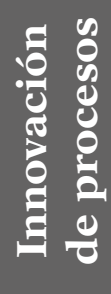 \\
\hline Redes de colaboración & 0.731 & & & & \\
\hline Capacidad de absorción & 0.089 & 0.757 & & & \\
\hline Adquisición tecnológica & 0.094 & 0.473 & 0.785 & & \\
\hline Innovación de producto & 0.091 & 0.377 & 0.297 & 0.796 & \\
\hline Proceso de innovación & 0.097 & 0.368 & 0.324 & 0.332 & 0.749 \\
\hline
\end{tabular}

Notas: Criterio de Fornell-Larcker: los valores diagonales (negrita) son la raíz cuadrada de la varianza compartida entre los constructos y sus medidas (VME). Para la validez discriminante, la raíz cuadrada del VME (negrita) es mayor que las correlaciones entre las otras variables latentes.

Fuente: Software AMOS.

\subsection{Modelo estructural}

Luego de verificar los modelos de medición, se estimó el modelo estructural.

La Tabla 3 presenta los coeficientes y valores p del modelo de investigación. 
Tabla 3. Resultados del modelo estructural

\begin{tabular}{|c|c|r|r|}
\hline Hipótesis & Relación & Coeficiente de trayectoria & \multicolumn{1}{|c|}{ Valor p } \\
\hline H1 & CN -> ACAP & $0.721 * * *$ & 0.001 \\
\hline H2 & ACAP - ProdI & $0.461 * * *$ & 0.001 \\
\hline H3 & ACAP -> ProcI & $0.251 * * *$ & 0.001 \\
\hline H4 & TECH -> ProdI & $0.201 *$ & 0.060 \\
\hline H5 & TECH ->ProcI & $0.251 * * *$ & 0.001 \\
\hline H6 & ProcI ->ProdI & $0.695 * * *$ & 0.001 \\
\hline
\end{tabular}

$\mathrm{CN}$ = Redes de colaboración; ACAP = capacidad de absorción; TECH = Adquisición tecnológica; ProdI = Innovación de producto; ProcI = Innovación de procesos.

Nota: n.s. = insignificante; * $\mathrm{p} \leq 0.1 ; * * \mathrm{p} \leq 0.05, * * * \mathrm{p} \leq 0.01, * * * * \mathrm{p} \leq 0.001$.

Fuente: Software AMOS.

Las variables de control se muestran en las Tablas 4 y 5.

Tabla 4. Variables de control para la innovación en producto

\begin{tabular}{|l|r|r|}
\cline { 2 - 3 } \multicolumn{1}{c|}{} & \multicolumn{1}{c|}{ Coef. } & \multicolumn{1}{c|}{ Val $\mathbf{p}$} \\
\hline Tamaño de la empresa & -0.137 & 0.006 \\
\hline Edad de la empresa & 0.050 & 0.258 \\
\hline
\end{tabular}

Fuente: Software AMOS.

Tabla 5. Variables de control para la innovación de procesos

\begin{tabular}{|l|r|r|}
\multicolumn{1}{l|}{} & \multicolumn{1}{c|}{ Coef. } & \multicolumn{1}{c|}{ Val p } \\
\hline Tamaño de la empresa & -0.085 & 0.030 \\
\hline Edad de la empresa & -0.045 & 0.194 \\
\hline
\end{tabular}

Fuente: Software AMOS.

Se puede observar que existe una relación pequeña, negativa y estadísticamente significativa entre el tamaño de la empresa y la innovación. En otras palabras, las empresas pequeñas tienen más probabilidades de innovar. Estos resultados coinciden con Lin et al. (2019), quienes señalan que las pequeñas empresas están más capacitadas para desarrollar innovaciones porque son más flexibles e independientes.

El modelo también cumple con el índice de bondad de ajuste, según los siguientes indicadores: CMIN / df, GFI, TLI, CFI y RMSEA. Los valores obtenidos muestran el ajuste adecuado del modelo de investigación (Tabla 6) (Singla et al., 2018).

Tabla 6. Resumen de ajuste del modelo

\begin{tabular}{|c|r|}
\hline Indicadores & \multicolumn{2}{|c|}{ Modelo estructural } \\
\hline CMIN/DF & 1.967 \\
\hline CFI & 0.962 \\
\hline NFI & 0.927 \\
\hline RFI & 0.903 \\
\hline TLI & 0.950 \\
\hline IFI & 0.963 \\
\hline RMSEA & 0.030 \\
\hline
\end{tabular}

Fuente: Software AMOS. 


\subsection{Análisis de la mediación}

Cuando se analizó la capacidad de absorción, se tomaron ciertas medidas para confirmar si era una variable mediadora y qué tipo de efecto tenía. Hair et al. (2014) demuestran que la mediación está presente cuando una variable mediadora es capaz de absorber de alguna manera cualquier efecto que pueda tener una construcción exógena (en el caso de variables independientes) o endógena (en el caso de variables dependientes). La varianza explicada (VE) determina hasta qué punto el proceso de mediación explica la varianza de la variable dependiente. Si la VE es menor al $20 \%$, se puede concluir que no hay mediación, y una VE mayor al $20 \%$ y menor al $80 \%$ indicaría mediación parcial (Hair et al., 2016). Un VE de más del $80 \%$ indica una mediación completa. El VCE es la relación entre el efecto indirecto $(0,191)$ y el efecto total $(0,306)$ para la capacidad de absorción entre redes de colaboración e innovación de producto y la relación entre el efecto indirecto $(0,119)$ y el efecto total $(0,296)$ para la capacidad de absorción. entre redes de colaboración e innovación de procesos, obteniendo $62 \%$ y $40 \%$, respectivamente. Por tanto, la mediación parcial de la capacidad absorbente está presente para ambas relaciones.

\section{Tabla 7. Prueba de mediación}

\begin{tabular}{|l|l|l|l|l|l|l|}
\hline Hipótesis & Influencia & $\begin{array}{c}\text { Efecto } \\
\text { directo } \\
(\text { valor p) }\end{array}$ & $\begin{array}{c}\text { Efecto } \\
\text { indirecto } \\
(\text { valor p) }\end{array}$ & $\begin{array}{c}\text { Efecto } \\
\text { total }\end{array}$ & $\begin{array}{c}\text { VC } \\
(\%)\end{array}$ & Interpretación \\
\hline H7a & $\begin{array}{l}\text { CN>ACAP> }> \\
\text { ProdI }\end{array}$ & $\begin{array}{l}0.115 * * \\
(0.014)\end{array}$ & $\begin{array}{l}0.191 * * \\
(0.014)\end{array}$ & $\begin{array}{l}0.306 * * \\
(0.014)\end{array}$ & $62 \%$ & Mediación parcial \\
\hline H7b & CN>ACAP> $>$ & $\begin{array}{l}0.177 * * * \\
(0.001)\end{array}$ & $\begin{array}{l}0.119 * * * \\
(0.001)\end{array}$ & $\begin{array}{l}0.296 * * * \\
(0.001)\end{array}$ & $40 \%$ & Mediación parcial \\
\hline
\end{tabular}

Nota: VC = varianza considerada; n.s. = insignificante; $* \mathrm{p} \leq 0,1 ; * \mathrm{p} \leq 0.05, * * * \mathrm{p} \leq 0.01, * * * * \mathrm{p} \leq 0.001$ significativo. VAF> $80 \%$ indica mediación total, $20 \% \leq \mathrm{VAF} \leq 80 \%$ indica mediación parcial y VAF $<20 \%$ indica que no hay mediación. Fuente: software SMART PLS, cálculos de los autores.

\section{Discusión y conclusiones}

Este estudio aporta a la literatura sobre innovación que establece que las empresas vinculadas a redes de colaboración obtienen conocimiento a través de ellas, aplicando el enfoque de innovación abierta inbound; este conocimiento, procesado a través de la capacidad de absorción de la empresa, mejorará su capacidad de innovación. Los resultados muestran que las redes de colaboración están relacionadas con la capacidad de absorción en empresas de tecnología baja y media-baja; los resultados obtenidos coinciden con los de Tsai (2001) y Hurmelinna-Laukkanen et al. (2012).

La literatura reconoce que la capacidad de absorción es uno de los determinantes de la innovación de productos. La identificación de la información externa y su proceso de asimilación logrado a través de la capacidad de absorción permiten a la empresa estar mejor capacitada para introducir nuevos productos en el mercado, adaptándose a las demandas de sus clientes. Este resultado coincide con los hallazgos de Murovec y Prodan (2008) y Martinez-Senra et al. (2015), quienes encontraron que las empresas con niveles más altos de capacidad de absorción son más capaces de desarrollar innovaciones de productos.

Numerosos estudios encuentran que las empresas desarrollan innovaciones en procesos a través de la adquisición de maquinaria y equipos, pero también es necesa- 
rio mencionar que el desarrollo de la capacidad de absorción permitirá a la empresa asimilar el conocimiento externo que proviene de proveedores, consultores, universidades y centros de investigación, lo que hará que la empresa desarrolle su capacidad de innovación y, por tanto, innovaciones de procesos. Estos resultados concuerdan con los obtenidos por Murovec y Prodan (2008) y Del Carpio y Miralles (2018), quienes encontraron que las empresas que desarrollan capacidad de absorción son más capaces de implementar innovaciones de proceso.

Las empresas de manufactura de baja o media-baja tecnología que invierten en maquinaria, hardware y software son más capaces de realizar innovaciones de producto, aunque, en gran medida, estas adquisiciones tecnológicas llevan a las empresas a realizar innovación de procesos y esta favorece la innovación de productos. Los resultados obtenidos coinciden con los de Vaona y Pianta (2008), Goedhuys y Veugelers (2012) y Pellegrino et al. (2012). En otras palabras, existe una relación positiva entre la adquisición de maquinaria, hardware y software y la innovación en productos y procesos.

Asimismo, las empresas de baja y media-baja intensidad tecnológica realizan innovaciones de producto con el fin de mantener o incrementar su posición en el mercado o tener clientes más satisfechos, mientras que realizan innovaciones de proceso para reducir sus costos operativos o ser más eficientes, lo que permite que estas empresas sean más capaces de desarrollar innovaciones en productos. Estos resultados coinciden con los obtenidos por Gunday et al. (2011) y Roldan y Bastos (2019).

En cuanto al papel mediador de la capacidad de absorción en la relación entre redes de colaboración e innovación tecnológica, en este estudio, la capacidad de absorción media parcialmente; sin embargo, esta mediación parcial se encuentra en niveles altos, cercanos a la mediación total, lo que coincide con los hallazgos de Tsai (2001) y Kostopoulos et al. (2011). La excepción es que, en estos estudios, la variable dependiente es la capacidad de innovación. En tanto, en el caso de la innovación de procesos, el papel mediador de la capacidad de absorción es solo parcial.

En cuanto a las variables de control, el tamaño de la empresa tiene una relación negativa y estadísticamente significativa con la innovación de productos. Este resultado parece contradecir a Tsai (2001), quien encontró que las grandes empresas tienen más recursos para realizar más innovaciones, pero, por otro lado, concuerda con Laforet (2008), quien indica que las pequeñas empresas de baja tecnología son más creativas al introducir nuevos productos en el mercado.

Se ha verificado empíricamente que las empresas de manufactura de baja tecnología, que participaron en la encuesta de innovación en 2015 y se conectaron con redes de mercado, tenían más probabilidades de desarrollar innovaciones de productos y procesos. Como empresas conectadas con redes institucionales, era más probable que desarrollaran innovaciones en los procesos.

Esta investigación ha contribuido a la literatura sobre innovación tecnológica realizada por empresas de baja y media-baja tecnología en una economía emergente —en este caso, Perú - analizando cómo estas empresas se conectan con redes de mercado e institucionales para poder desarrollar innovaciones tecnologías.

Los resultados de este estudio permiten identificar algunas implicaciones prácticas. Los gerentes de empresas de baja tecnología deben fortalecer las conexiones con clientes, proveedores y competidores y, al mismo tiempo, con universidades e institutos de investigación con la intención de desarrollar un mayor número de innovaciones tecnológicas.

Este estudio no está exento de limitaciones, que son las siguientes:

La primera limitación se refiere a cómo ha utilizado la base de datos que se obtuvo de la encuesta nacional de innovación de la industria manufacturera en Perú de 2015. Como estudio transversal, enfrenta dos problemas: el sesgo generado por el 
hecho de que una sola persona de la firma respondió a los cuestionarios y el hecho de que este tipo de estudio no permite establecer una relación causal entre constructos (Rindfleisch et al., 2008). Se sugiere que se realicen estudios longitudinales. Otra opción serían los estudios comparativos utilizando las encuestas de innovación realizadas en otros países de América Latina.

La segunda limitación tiene que ver con cómo se han medido los constructos de las redes institucionales y de mercado; estos constructos reflejan conexiones con clientes, proveedores o competidores e institutos de investigación públicos o privados. Se sugiere que se realicen estudios que identifiquen conexiones específicas, por ejemplo, conexiones con clientes, proveedores o universidades, y cómo estas relaciones favorecen individualmente el desarrollo de innovaciones de productos o procesos.

\section{Declaración de conflictos de intereses}

Los autores declararon no tener ningún conflicto de intereses potencial con respecto a la investigación, autoría y/o publicación del artículo.

\section{Referencias}

Agramunt, L.F., Berbel-Pineda, J.M. Capobianco-Uriarte, M.M., \& Casado-Belmonte, M.P. (2020). Review on the Relationship of Absorptive Capacity with Interorganizational Networks and the Internationalization Process. Complexity. https://doi.org/10.1155/2020/7604579

Aliasghar, O. Sadeghi, A. \& Rose E. L. (2020) Process innovation in small- and medium-sized enterprises: The critical roles of external knowledge sourcing and absorptive capacity, Journal of Small Business Management, https://doi.org/10.1080/00472778.2020.1844491

Baker, W.E., Grinstein, A., \& Harmancioglu, N. (2016). Whose innovation performance benefits more from external networks: Entrepreneurial or conservative firms? Journal of Product Innovation Management, 33(1), 104-120. https://doi.org/10.1111/jpim.12263

Ballot, G., Fakhfakh, F., Galia, F., \& Salter, A. (2015). The fateful triangle: Complementarities in performance between product, process and organizational innovation in France and the UK, Research Policy, 44(1), 217-232. https://doi.org/10.1016/j.respol.2014.07.003

Bayona-Saez, C., Cruz-Cázares, C., García-Marco, T., \& Sánchez-García, M. (2017). Open innovation in the food and beverage industry. Management Decision, 55(3), 526-546. https://doi. org/10.1108/md-04-2016-0213

Breznik, L., \& Hisrich, R.D (2014). Dynamic capabilities vs. innovation capability: are they related? Journal of Small Business and Enterprise Development, 21(3), 368-384. https://doi.org/10.1108/ JSBED-02-2014-0018

Caloghirou, Y., Kastelli, I., \& Tsakanikas, A. (2004). Internal capability and external knowledge sources: Complements or substitutes for innovative performance? Technovation, 24(1), 29-39. https://doi.org/10.1016/s0166-4972(02)00051-2

Camisón, C., \& Villar-López, A. (2014). Organizational innovation as an enabler of technological innovation capabilities and firm performance. Journal of Business Research, 67(1), 2891-2902. https://doi.org/10.1016/j.jbusres.2012.06.004

Cantner, U., \& Joel, K. (2011). Network position, absorptive capacity and firm success. IUP Journal of Knowledge Management, 9(1), 57-83.

Coccia, M. (2017). Sources of technological innovation: Radical and incremental innovation problem-driven to support competitive advantage of firms. Technology Analysis $\mathcal{B}$ Strategic Management, 29(9), 1048-1061. https://doi.org/10.1080/09537325.2016.1268682

Cohen, W.M., \& Levinthal, D.A. (1990). Absorptive capacity: A new perspective on learning and innovation. Administrative Science Quarterly, 35(1), 128-152. https://doi.org/10.2307/2393553

Conte, A., \& Vivarelli, M. (2014). Succeeding in innovation: Key insights on the role of R\&D and technological acquisition drawn from company data. Empirical Economics, 47(4), 1317-1340. https://doi.org/10.1007/s00181-013-0779-1 
Coronado-Medina, A., Arias-Pérez, J., \& Perdomo-Charry, G. (2020). Fostering product innovation through digital transformation and absorptive capacity. International Journal of Innovation and Technology Management (IJITM), 17(06), 1-23. https://doi.org/10.1142/S0219877020500406

Del Carpio-Gallegos, J.F., \& Miralles-Torner, F. (2018). Absorptive capacity and innovation in lowtech companies in emerging economies. Journal of Technology Management $\mathbb{E}$ Innovation, 13(2), 3-11. https://doi.org/10.4067/s0718-27242018000200003

Del Carpio, J. F., \& Miralles, F. (2019). Analyzing technological innovation in low and medium-low tech Peruvian manufacturing companies. Contaduría y Administración, 64(4), 1-22. https://doi. org/10.22201/fca.24488410e.2018.1830

Doran, J. (2012). Are differing forms of innovation complements or substitutes? European Journal of Innovation Management, 15(3), 351-371. https://doi.org/10.1108/14601061211243675

Eisenhardt, K., \& Martin, J. (2000). Dynamic capabilities: What are they? Strategic Management Journal, 21(10/11), 1105-11(22). https://doi.org/10.1002/1097-0266(200010/11)21:10/11<1105::aid -smj133>3.0.co;2-e

Escribano, A., Fosfuri, A., \& Tribó, J.A. (2009). Managing external knowledge flows: The moderating role of absorptive capacity. Research Policy, 38(1), 96-105. https://doi.org/10.1016/j.respol.2008.10.022

Filippetti, A. (2011). Innovation modes and design as a source of innovation: A firm-level analysis. European Journal of Innovation Management, 14(1), 5-26. https://doi.org/10.1108/14601061111104670

Fornell, C., \& Larcker, D.F. (1981). Structural equation models with unobservable variables and measurement error: Algebra and statistics. Journal of Marketing Research, 18(1), 39-50. https:// doi.org/10.2307/3150980

Frank, A.G., Cortimiglia, M.N., Ribeiro, J.L.D., \& de Oliveira, L.S. (2016). The effect of innovation activities on innovation outputs in the Brazilian industry: Market-orientation vs. technology-acquisition strategies. Research Policy, 45(3), 577-592. https://doi.org/10.1016/j.respol.2015.11.011

Frigon, A., Doloreux, D., \& Shearmur, R. (2020). Drivers of eco-innovation and conventional innovation in the Canadian wine industry. Journal of Cleaner Production, 275, 124115. https://doi. org/10.1016/j.jclepro.2020.124115

Goedhuys, M., \& Veugelers, R. (2012). Innovation strategies, process and product innovations and growth: Firm-level evidence from Brazil. Structural Change and Economic Dynamics, 23(4), 516529. https://doi.org/10.1016/j.strueco.2011.01.004

Goedhuys, M., Janz, N., \& Mohnen, P. (2014). Knowledge-based productivity in "low-tech" industries: Evidence from firms in developing countries. Industrial and Corporate Change, 23(1), 1-23. https://doi.org/10.1093/icc/dtt006

Grimpe, C., \& Sofka, W. (2009). Search patterns and absorptive capacity: Low-and high-technology sectors in European countries. Research Policy, 38(3), 495-506. https://doi.org/10.1016/j. respol.2008.10.006

Gronum, S.V. (2012). The role of networks in small and medium-sized enterprise innovation and firm performance. Journal of Small Business Management, 50(2), 257-282. https://doi.org/10.1111/ j.1540-627x.2012.00353.x

Gunday, G., Ulusoy, G., Kilic, K., \& Alpkan, L. (2011). Effects of innovation types on firm performance. International Journal of production economics, 133(2), 662-676. https://doi.org/10.1016/j. ijpe.2011.05.014

Hair Jr., J.F., Sarstedt, M., Hopkins, L., \& Kuppelwieser, V.G. (2014). Partial least squares structural equation modeling (PLS-SEM) An emerging tool in business research. European Business Review, 26(2), 106-121. https://doi.org/10.1108/ebr-10-2013-0128

Hair Jr., J., Hult, T., Ringle, C., \& Sarstedt, M. (2016). A primer on partial least squares structural equation modeling (PLS-SEM). Sage.

Hassan, M. U., Shaukat, S., Nawaz, M. S., \& Naz, S. (2013). Effects of innovation types on firm performance: An empirical study on Pakistan's manufacturing sector. Pakistan Journal of Commerce and Social Sciences, 7(2), 243-262. 
Hervas-Oliver, J.L., Boronat-Moll, C., \& Sempere-Ripoll, F. (2016). On process innovation capabilities in SMEs: A taxonomy of process-oriented innovative SMEs. Journal of Small Business Management, 54, 113-134. https://doi.org/10.1111/jsbm.12293

Huang, F., \& Rice, J. L. (2012). Openness in product and process innovation. International Journal of Innovation Management, 16(4), 1-24. https://doi.org/10.1142/s1363919612003812

Hurmelinna-Laukkanen, P., Olander, H., Blomqvist, K., \& Panfilii, V. (2012). Orchestrating R\&D networks: Absorptive capacity, network stability, and innovation appropriability. European Management Journal, 30(6), 552-563. https://doi.org/10.1016/j.emj.2012.03.002

Jespersen, K., Rigamonti, D., Jensen, M. B., \& Bysted, R. (2018). Analysis of SMEs partner proximity preferences for process innovation. Small Business Economics, 51(4), 879-904. https://doi. org/10.1007/s11187-017-9969-0

King, A.A., \& Baatartogtokh, B. (2015). How useful is the theory of disruptive innovation? MIT Sloan Management Review, 57(1), 77. https://bit.ly/3zlDdV9

Kostopoulos, K., Papalexandris, A., Papachroni, M., \& Ioannou, G. (2011). Absorptive capacity, innovation, and financial performance. Journal of Business Research, 64(12), 1335-1343. https:// doi.org/10.1016/j.jbusres.2010.12.005

Laforet, S. (2008). Size, strategic, and market orientation affects on innovation. Journal of Business Research, 61(7), 753-764. https://doi.org/10.1016/j.jbusres.2007.08.002

Lai, Y.L., Hsu, M.S., Lin, F.J., Chen, Y.M., \& Lin, Y.H. (2014). The effects of industry cluster knowledge management on innovation performance. Journal of Business Research, 67(5), 734-739. https://doi.org/10.1016/j.jbusres.2013.11.036

Liao, Y., \& Barnes, J. (2015). Knowledge acquisition and product innovation flexibility in SMEs. Business Process Management Journal, 21(6), 1257-1278. https://doi.org/10.1108/bpmj-05-2014-0039

Liao, S.H., Wu, C.C., Hu, D.C., \& Tsui, K.A. (2010). Relationships between knowledge acquisition, absorptive capacity and innovation capability: an empirical study on Taiwan's financial and manufacturing industries. Journal of Information Science, 36(1), 19-35. https://doi. org/10.1177/0165551509340362

Lin, W. L., Cheah, J. H., Azali, M., Ho, J. A., \& Yip, N. (2019). Does firm size matter? Evidence on the impact of the green innovation strategy on corporate financial performance in the automotive sector. Journal of Cleaner Production, 229, 974-988. https://doi.org/10.1016/j.jclepro.2019.04.214

Love, J. H., \& Roper, S. (2015). SME innovation, exporting and growth: A review of existing evidence. International Small Business Journal, 33(1), 28-48. https://doi.org/10.1177/0266242614550190

Marrocu, E., Paci, R., \& Usai, S. (2013). Proximity, networking and knowledge production in Europe: What lessons for innovation policy? Technological Forecasting and Social Change, 80(8), 14841498. https://doi.org/10.1016/j.techfore.2013.03.004

Martín-de Castro, G. (2015). Knowledge management and innovation in knowledge-based and high-tech industrial markets: The role of openness and absorptive capacity. Industrial Marketing Management, 47, 143-146. https://doi.org/10.1016/j.indmarman.2015.02.032

Martinez-Senra, A. I., Quintas, M. A., Sartal, A., \& Vázquez, X. H. (2015). How can firms' basic research turn into product innovation? The role of absorptive capacity and industry appropriability. IEEE Transactions on Engineering Management, 62(2), 205-216. https://doi.org/10.1109/ tem.2014.2386614

Martino, G., Rossetti, E., Marchini, A., \& Frascarelli, A. (2017). Process innovation in milling stage in olive oil sector: Evidence from an empirical analysis in Umbria (Italy). British Food Journal, 119(8), 1748-1765. https://doi.org/10.1108/bfj-12-2016-0585

Medrano, L.A., \& Muñoz-Navarro, R. (2017). Aproximación conceptual y práctica a los modelos de ecuaciones estructurales. Revista Digital de Investigación en Docencia Universitaria, 11(1), 219-239. https://doi.org/10.19083/ridu.11.486

Moilanen, M., Østbye, S., \& Woll, K. (2014). Non-R\&D SMEs: External knowledge, absorptive capacity and product innovation. Small Business Economics, 43(2), 447-462. https://doi. org/10.1007/s11187-014-9545-9 
Murmura, F., Bravi, L., \& Santos, G. (2021). Sustainable Process and Product Innovation in the Eyewear Sector: The Role of Industry 4.0 Enabling Technologies. Sustainability, 13(1), 365. https://doi.org/ 10.3390/su13010365

Murovec, N., \& Prodan, I. (2008). The influence of organizational absorptive capacity on product and process innovation. Organizacija, 41(2), 43-49. https://doi.org/10.2478/v10051-008-0005-7

Najafi-Tavani, S., Sharifi, H., \& Ismail, H.S. (2013). A study of contingency relationships between supplier involvement, absorptive capacity and agile product innovation. International Journal of Operations $\mathcal{E}$ Production Management, 34(1), 65-92. https://doi.org/10.1108/ijopm-09-2011-0331

Nicotra, M., Romano, M., \& Del Giudice, M. (2014). The evolution dynamic of a cluster knowledge network: The role of firms' absorptive capacity. Journal of the Knowledge Economy, 5(1), 70-93. https://doi.org/10.1007/s13132-012-0140-5

Nieto, M.J., \& Santamaría, L. (2007). The importance of diverse collaborative networks for the novelty of product innovation. Technovation, 27(6-7), 367-377. https://doi.org/10.1016/j.technovation.2006.10.001

Pellegrino, G., Piva, M., \& Vivarelli, M. (2012). Young firms and innovation: A microeconometric analysis. Structural Change and Economic Dynamics, 23(4), 329-340 https://doi.org/10.1016/j.strueco.2011.10.003

Piening, E.P., \& Salge, T.O. (2015). Understanding the antecedents, contingencies, and performance implications of process innovation: A dynamic capabilities perspective. Journal of Product Innovation Management, 32(1), 80-97. https://doi.org/10.1111/jpim.12225

Ponce-Espinosa, G., Espinoza, D.L., Ríos-Zaruma, J., \& Tapia-Carreño, K.G. (2017). Organizational capacities to generate value: analysis of industrial sector. Retos. Revista de Ciencias de la Administración y Economía, 7(13), 271-290. http://dx.doi.org/10.17163/ret.n13.2017.09

Reichstein, T., \& Salter, A. (2006). Investigating the sources of process innovation among UK manufacturing firms. Industrial and Corporate Change, 15(4), 653-682. https://doi.org/10.1093/icc/ dt1014

Rindfleisch, A., Malter, A. J., Ganesan, S., \& Moorman, C. (2008). Cross-sectional versus longitudinal survey research: Concepts, findings, and guidelines. Journal of Marketing Research, 45(3), 261-279. https://doi.org/10.1509/jmkr.45.3.261

Ritala, P., \& Hurmelinna-Laukkanen, P. (2013). Incremental and radical innovation in coopetition-The role of absorptive capacity and appropriability. Journal of Product Innovation Management, 30(1), 154-169. https://doi.org/10.1111/j.1540-5885.2012.00956.x

Roldan, L.B. \& Bastos, W.N. (2019). Analysis of relations between the types of innovations and performance in technological parks. SBIR-Small Business International Review, 3(1), 36-48. https:// doi.org/10.26784/sbir.v3i1.172

Romero-Hidalgo, O.M., Jaramillo-Paredes, M.F., Aguirre-Benalcázar, M.C., \& Quezada-Abad, C.J. (2021). Modelos gerenciales en las medianas empresas de Ecuador y Perú . Revista de la Universidad del Zulia, 12(32), 374-399. https://doi.org/10.46925//rdluz.32.23

Rouvinen, P. (2002). Characteristics of product and process innovators: Some evidence from the Finnish innovation survey. Applied Economics Letters, 9(9), 575-580. https://doi. org/10.1080/13504850110108102

Saenz, M.J., Revilla, E., \& Knoppen, D. (2014). Absorptive capacity in buyer-supplier relationships: empirical evidence of its mediating role. Journal of Supply Chain Management, 50(2), 18-40. https://doi.org/10.1111/jscm.12020

Schoenmakers, W., \& Duysters, G. (2006). Learning in strategic technology alliances. Technology Analysis $\mathcal{E}$ Strategic Management, 18(2), 245-264. https://doi.org/10.1080/09537320600624162

Scuotto, V., Del Giudice, M., \& Carayannis, E.G. (2017). The effect of social networking sites and absorptive capacity on SMES' innovation performance. The Journal of Technology Transfer, 42(2), 409-424. https://doi.org/10.1007/s10961-016-9517-0

Segarra-Blasco, A., \& Arauzo-Carod, J. M. (2008). Sources of innovation and industry-university interaction: Evidence from Spanish firms. Research Policy, 37(8), 1283-1295. https://doi.or$\mathrm{g} / 10.1016 / \mathrm{j}$. respol.2008.05.003 
Singla, A., Ahuja, I.S., \& Sethi, A. (2018). Validation of technology push strategies for achieving sustainable development in manufacturing organizations through structural equation modeling. World Journal of Science, Technology and Sustainable Development, 15(1), 72-93. https://doi. org/10.1108/WJSTSD-08-2017-0022

Spithoven, A., Clarysse, B., \& Knockaert, M. (2010). Building absorptive capacity to organise inbound open innovation in traditional industries. Technovation, 30(2), 130-141. https://doi.or$\mathrm{g} / 10.1016 / \mathrm{j}$.technovation.2009.08.004

Tsai, W. (2001). Knowledge transfer in intraorganizational networks: Effects of network position and absorptive capacity on business unit innovation and performance. Academy of Management Journal, 44(5), 996-1004. https://bit.ly/3gen0d8

Tzokas, N., Kim, Y.A., Akbar, H., \& Al-Dajani, H. (2015). Absorptive capacity and performance: The role of customer relationship and technological capabilities in high-tech SMEs. Industrial Marketing Management, 47, 134-142. https://doi.org/10.1016/j.indmarman.2015.02.033

Vaona, A., \& Pianta, M. (2008). Firm size and innovation in European manufacturing. Small Business Economics, 30(3), 283-299. https://doi.org/10.1007/s11187-006-9043-9

Zapata-Rotundo, G.J., \& Hernández-Arias, A. (2018). Capacidad de absorción: revisión de la literatura y un modelo de sus determinantes. Retos Revista de Ciencias de la Administración y Economía, 8(16), 121-140. https://doi.org/10.17163/ret.n16.2018.09

Zhou, K.Z., \& Wu, F. (2010). Technological capability, strategic flexibility, and product innovation. Strategic Management Journal, 31(5), 547-561. https://doi.org/10.1002/smj.830 